\title{
Silent Sinus Syndrome: A Case Report and Review of Literature
}

\author{
Marina Saldanha, Vadisha Bhat, B Satheesh Kumar Bhandary, Sebin Tom Scaria
}

\begin{abstract}
Silent sinus syndrome is a rare condition affecting the maxillary sinus, resulting in spontaneous collapse of the sinus walls causing facial asymmetry and unilateral enophthalmos. The exact etiology of this condition is unknown. We report a case of a 20 -year-old male presented with facial asymmetry with depression of right cheek associated with heaviness over right cheek, without any disturbance of vision or eye movement. Computed tomography (CT) scan of the paranasal sinus showed a relatively smaller and opacified right maxillary sinus with inward retraction of the roof, medial and posterolateral walls. He underwent endoscopic middle meatal antrostomy. On follow-up, patient is free of symptoms and the CT scan showed well-ventilated sinuses with arrest of the disease progression.
\end{abstract}

Keywords: Silent sinus syndrome, Maxillary sinus, Endoscopic middle meatal antrostomy.

How to cite this article: Saldanha M, Bhat V, Bhandary BSK, Scaria ST. Silent Sinus Syndrome: A Case Report and Review of Literature. Clin Rhinol An Int J 2013;6(3):144-148.

Source of support: Nil

Conflict of interest: None declared

\section{INTRODUCTION}

Silent sinus syndrome is a rare cause of facial asymmetry as a result of spontaneous collapse of maxillary sinus walls. ${ }^{1}$ Retraction of the superior wall of the maxillary sinus results in increase in the orbital volume and cause enophthalmos which is the commonest feature of presentation. The etiopathogenesis of the silent sinus syndrome is not well understood. But, the final common pathway causing this disease is the obstruction of maxillary sinus ostium, leading to a chronic hypoventilated state. ${ }^{2}$ However, the symptoms of chronic sinusitis are absent in most of the cases. ${ }^{3}$ Clinical diagnosis of silent sinus syndrome needs radiological confirmation. Computer tomography (CT) scan of paranasal sinus shows a fully developed opacified sinus with inward retraction of sinus walls into the lumen with associated loss of sinus volume. Maxillary infundibulum is occluded. ${ }^{2}$ Definitive treatment of silent sinus syndrome is creating a maxillary antrostomy, which can be achieved endoscopically. ${ }^{3}$ While most of the patients become asymptomatic when the sinus is ventilated, a few of them require reconstruction of orbital floor which can be achieved as a second stage procedure. ${ }^{4}$ Here, we report a case of a 20-year-old male presented with progressive depression of the right cheek without any symptoms related to the eye. Computer tomography scan showed features of silent sinus syndrome. He underwent endoscopic middle meatal antrostomy, which resulted in arrest of the disease progression.

\section{CASE REPORT}

A 20-year-old male presented with history of gradually progressive depression of the right cheek with heaviness over the right cheek and dull aching pain around the right eye of 6 months duration. There is no history of nasal discharge, nasal obstruction, epistaxis or facial trauma. There was no vision disturbance. He did not have any systemic diseases. Patient had history of frequent swimming and, before 2 years, he had noticed pain over the right cheek after diving, which improved later.

His general health state was good. There was asymmetry of the face caused by depression of the right cheek (Fig. 1). Ophthalmological evaluation revealed no abnormality in the vision or eye movement. Nasal cavity was normal. Computed Tomography scan of the paranasal sinus coronal and axial images showed relatively small right maxillary sinus with inward retraction of the roof, medial and posterolateral walls. Infundibulum was obstructed with complete opacification of sinus (Figs 2 and 3). Frontal, ethmoid and sphenoid sinuses of both sides were normal (Figs 4 to 6). A diagnosis of silent sinus syndrome was made. Patient underwent endoscopic middle meatal antrostomy (Fig. 7). Polypoid mucosa was cleared from the right maxillary sinus. Tissue sent for histopathological examination. Histological features were suggestive of inflammatory sinonasal polyp (Fig. 8). Patient is under

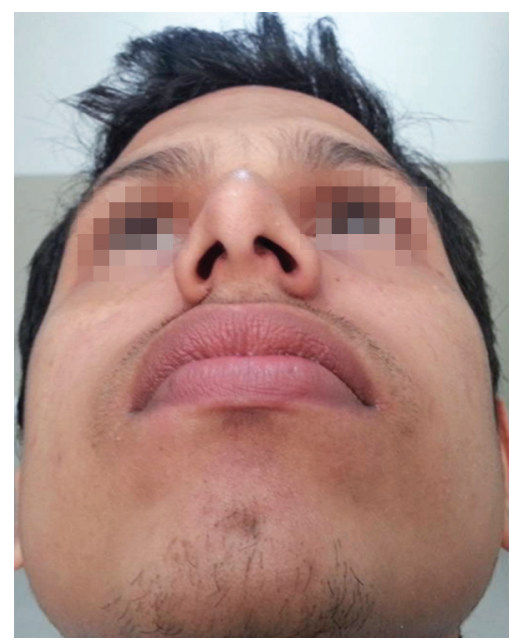

Fig. 1: Clinical photograph showing depression of the right cheek 


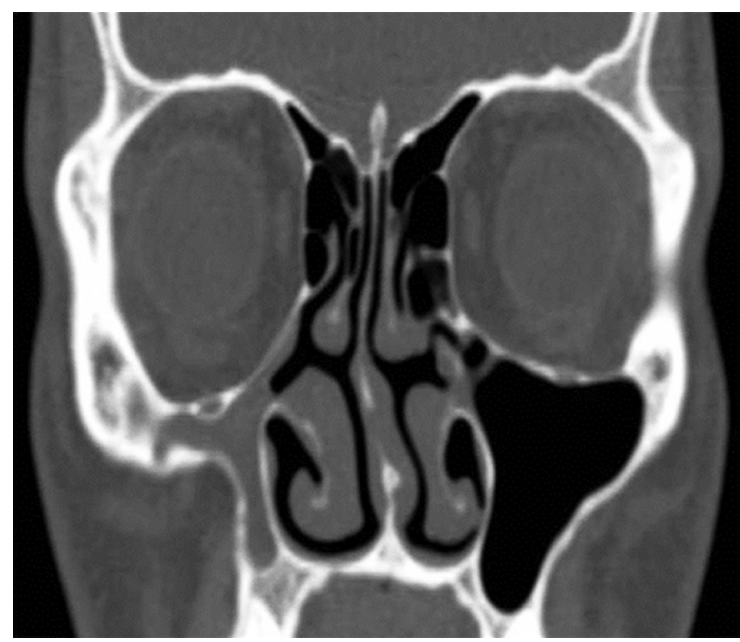

Fig. 2: CT scan of paranasal sinus - coronal view showing inward retraction of medial, posterolateral and superior walls of maxillary sinus with obstruction at infundibulum and complete opacification of right maxillary sinus

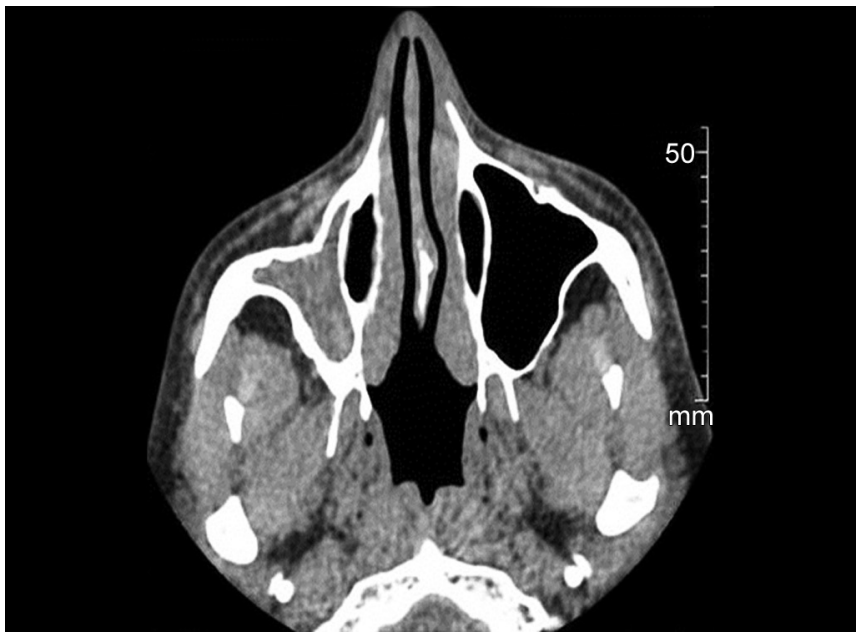

Fig. 3: CT scan of paranasal sinus - axial view showing inward retraction of anterior and posterolateral walls of maxillary sinus with complete opacification of right maxillary sinus

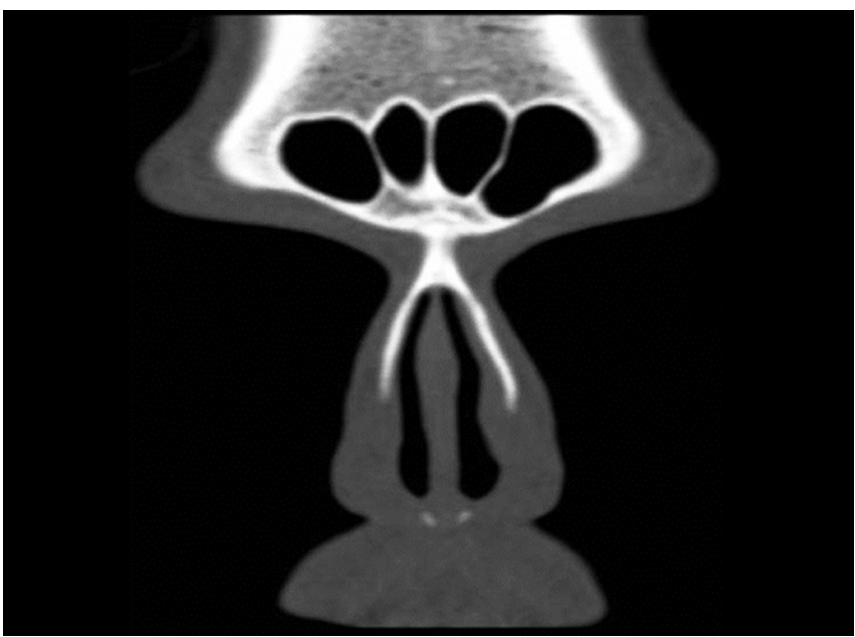

Fig. 4: CT scan of paranasal sinus - coronal view showing normal frontal sinus

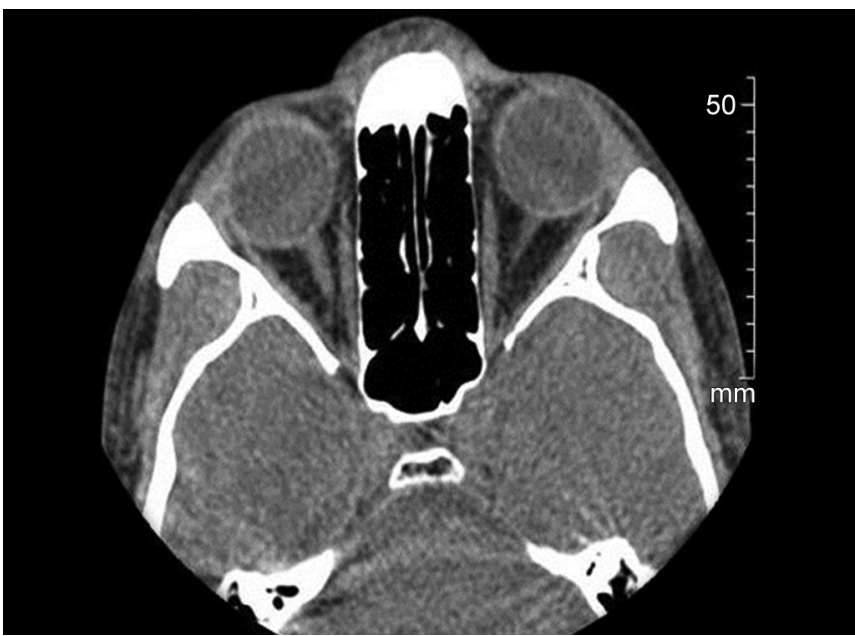

Fig. 5: CT scan of paranasal sinus - axial view showing normal ethmoid sinus

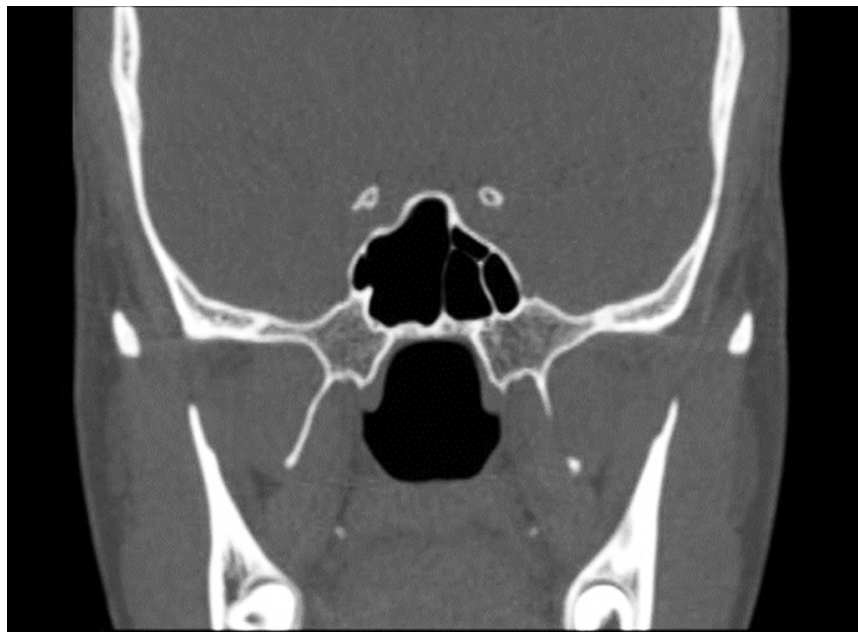

Fig. 6: CT scan of paranasal sinus - coronal view showing normal sphenoid sinus

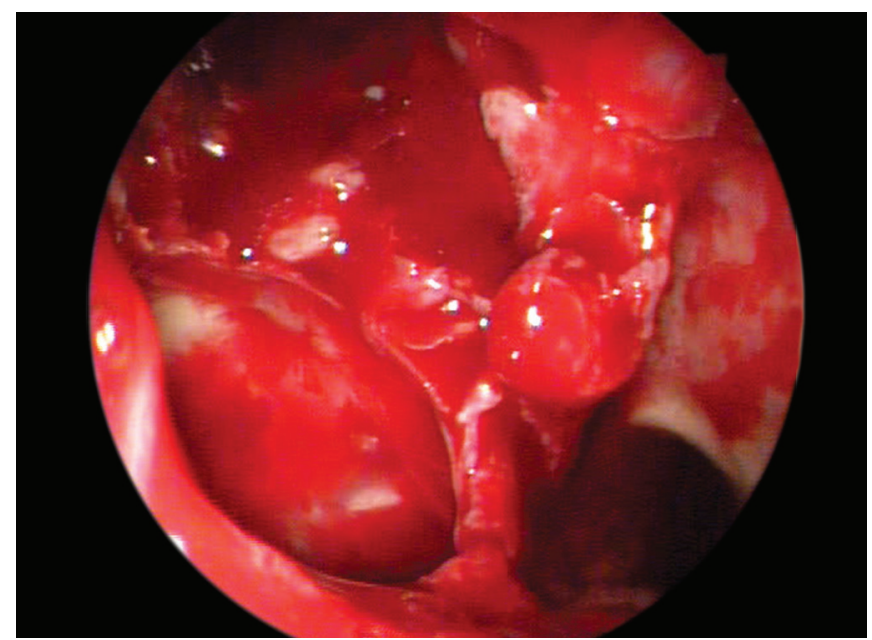

Fig. 7: Endoscopic middle meatal antrostomy 


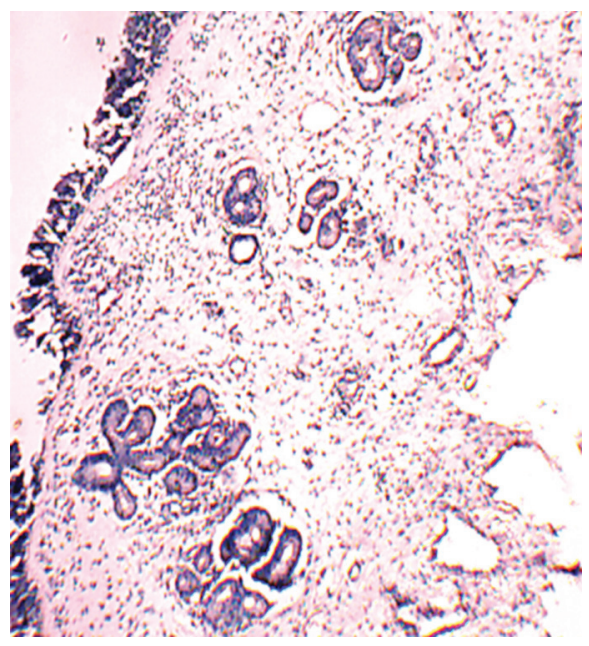

Fig. 8: Histopathology (H\&E stain) showing features of inflammatory sinonasal polyp

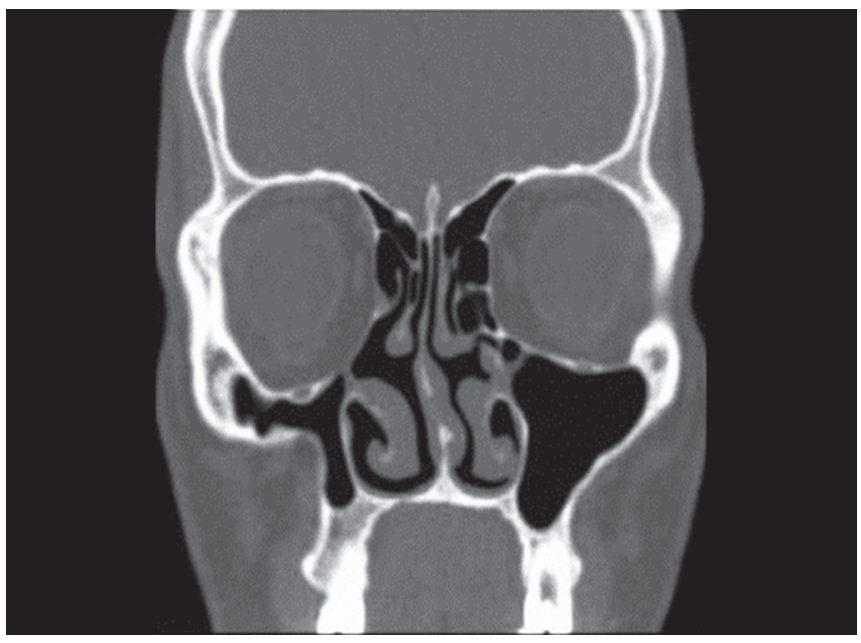

Fig. 9: CT scan of paranasal sinus, 3 months postoperative coronal view showing well-ventilated maxillary sinus

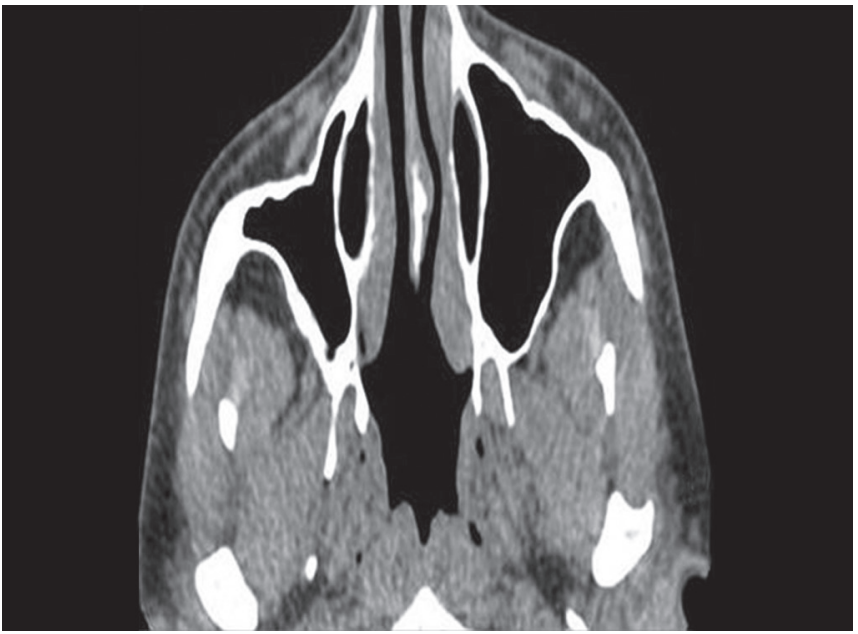

Fig. 10: CT scan of paranasal sinus axial, 3 months postoperative view showing well-ventilated maxillary sinus

regular follow-up. His heaviness over the cheek is here is no progression of the deformity in 3 months follow-up period. Computer tomography scan of paranasal sinuses after 3 months show well-ventilated right maxillary sinus with arrest of the disease process (Figs 9 and 10).

\section{DISCUSSION}

Silent sinus syndrome is a rare disease caused by chronic maxillary sinus atelectasis resulting in facial asymmetry and painless enophthalmos. The term silent sinus syndrome was coined by Soparkar et al in 1994. ${ }^{1}$ However, the description of chronic maxillary atelectasis was given by Montgomery et al in $1964 .{ }^{5}$ The condition has gained much attention due to the slowly progressive facial asymmetry and enophthalmos, in the absence of classic features of sinusitis, even though the radiological investigations reveal a completely opacified maxillary sinus. Being a rare condition, the literature on this disease is limited to case reports, with only few case series, the largest one being a review of 22 cases by Kass et al. ${ }^{6}$ This condition is seen more frequently in younger population between the third and fifth decade of life, without gender predilection. ${ }^{3}$ The clinical presentation of silent sinus syndrome is progressive facial asymmetry, enophthalmos and hypoglobus. The eye symptoms are due to the downward displacement of orbital floor. Symptoms related to sinusitis may be present but not a necessity. ${ }^{2}$ In our case, patient presented with facial asymmetry due to progressive depression of the right cheek and heaviness over the right cheek and pain around right eye, which is more likely due to underlying sinusitis. He did not have any symptoms pertaining to the eye. The term chronic maxillary atelectasis (CMA) refers to a persistent decrease in the sinus volume of the maxilla from inward bowing of the antral walls, which is often used interchangeably with silent sinus syndrome. However, the term CMA is used when it is associated with sinus symptoms. Brandt et al in 2008 did a systematic review of 105 reported cases of silent sinus syndrome, of which 55 contained the complete set of data, 27 of these met the diagnostic criteria of silent sinus syndrome and 48 could be diagnosed as chronic maxillary atelectasis, 23 of the case met the criteria for both conditions. They suggested abandoning the term silent sinus syndrome and recommended to use the term chronic maxillary atelectasis for all such cases. ${ }^{7}$

The etiopathogenesis of silent sinus syndrome is not clearly established. There are proponents of congenital theory, suggesting congenital hypoplasia of maxillary sinus with obstruction of the sinus ostium as the cause. ${ }^{1}$ However, now there is enough evidence to prove that this is an acquired condition. Hourany et al reported a case of silent sinus syndrome in an adult male who had a normal maxillary sinus in a CT scan done before 10 years for some other reason. ${ }^{8}$ Illner et al, in their series of five patients of silent sinus syndrome, reported fully developed maxillary 
sinus suggested by extension of the sinus laterally into malar eminence and inferiorly into the maxillary alveolar ridge. ${ }^{2}$ They opined opacified congenital hypoplastic maxillary sinus may be a less severe form of silent sinus syndrome with the smaller sinus volume and shorter, thicker sinus walls. Kruger et al suggested barotrauma as the possible etiology, in one of their case report of silent sinus syndrome seen in scuba divers. ${ }^{9}$ The patient in this report also had history of swimming and diving and, 2 years back, he had developed sudden onset of pain over the right cheek after diving, which improved later. Barotrauma while diving may be the etiology of maxillary sinus atelectasis in this case. The final pathway leading to silent sinus syndrome seems to be obstruction of ventilation and mucous drainage of the maxillary sinus which causes negative pressure in the sinus, thus leading to a chronic inflammatory state and osteolysis of the sinus walls. ${ }^{2}$ Negative intrasinus pressure has been demonstrated by Davidson et al. ${ }^{10}$ Levine and Mitra reported a case of maxillary sinus involution after endoscopic sinus surgery. ${ }^{11}$ Even in this case, they proposed ostiomeatal complex obstruction may be the likely cause for sinus involution. But surprisingly, the prevalence of maxillary sinus obstruction is very high compared to the rare incidence of silent sinus syndrome. ${ }^{8}$ Most of the reported cases of silent sinus syndrome involve maxillary sinus. In 2013, Naik et al reported a case of a silent sinus syndrome of frontal sinus caused by obstruction of sinus opening by a large type III Kuhn cell. ${ }^{12}$

Silent sinus syndrome produce characteristic features on CT scan of paranasal sinus. Both coronal and axial views are advised for complete evaluation of sinus anatomy and pathology. The imaging findings include inward retraction of the sinus walls into the sinus lumen, causing reduction of sinus volume. The affected sinus almost always had mucosal disease, resulting in partial or complete opacification of the sinus. Infundibulum is obstructed, which is supposed to be the igniting factor. Illner et al also noted the uncinate process to be laterally retracted with apposition of the uncinate process with the inferomedial orbital wall. ${ }^{2}$ The downward displacement of floor of the orbit causes resultant increase in orbital volume, causing enophthalmos. ${ }^{2}$ In our case, even though the CT scan showed downward displacement of orbital wall and increased volume of the right orbit, there was no disturbance of the vision or eye movement. This is unusual as most of the reported cases with orbital floor displacement had enophthalmos or hypoglobus. Choudhury and Marais et al reported a case of silent sinus syndrome wherein there was collapse of only medial wall of the sinus, resulting in volume loss. The patient did not have displacement of floor of orbit. They concluded this could be one of the early cases, which might later progress to frank silent sinus syndrome. ${ }^{3}$

The treatment of silent sinus syndrome is clearing the disease from the affected maxillary sinus and creating an outlet for mucous drainage from the obstructed sinus. Caldwell-Luc surgery and transconjunctival repair of the orbital floor were the procedures done in the past. ${ }^{3}$ However, now the procedure can be performed endoscopically by creating a middle meatal antrostomy. After surgery, the sinus configuration may remain unchanged, improve slightly or may revert to normal over time. ${ }^{2}$ But the disease process arrested preventing further deformity. Repair of the orbital floor may be done during the primary procedure or as a second stage procedure. As some patients' enophthalmos improve with middle meatal antrostomy alone, it is wiser to plan orbital floor correction for second stage, if there is no improvement.

\section{CONCLUSION}

Silent sinus syndrome is a rare disease of the maxillary sinus, characterized by facial asymmetry and painless enophthalmos, as a result of chronic hypoventilated maxillary sinus leading to collapse of the sinus walls into the lumen. Imaging in the form of CT scan of paranasal sinus plays a crucial rule in diagnosing this condition, as many of them do not have features of sinusitis. Barotrauma caused during diving might be a factor in the pathogenesis of maxillary atelectasis, though the final common pathway is obstruction at infundibulum causing negative intrasinus pressure. Endoscopic middle meatal antrostomy is the treatment of choice for silent sinus syndrome, which can lead to reversal of sinus architecture or at least arrests the disease progression.

\section{REFERENCES}

1. Soparkar CNS, Patrinely JR, Cuaycong MJ, et al. The silent sinus syndrome: a cause of spontaneous enophthalmos. Ophthalmology 1994;101:772-778.

2. Illner A, Davidson HC, Harnsberger HR, Hoffman J. The silent sinus syndrome: clinical and radiologic findings. AJR 2002; 178:503-506

3. Choudhury N, Marais J. The silent sinus syndrome: a clinical review. Clinical Rhinology: An International Journal 2010;3(2):69-72.

4. Thomas RD, Graham SM, Carter KD, Nerad JA. Management of the orbital floor in silent sinus syndrome. Am J Rhinol 2003 Mar-Apr;17(2):97-100.

5. Montgomery WW. Mucocele of the maxillary sinus causing enophthalmos. Eye Ear Nose Throat Monthly 1964 May;43: 41-44. 
6. Kass ES, Salman S, Rubin PAD, Weber AL, MontgomeryWW. Chronic maxillary atelectasis. Ann Otol Rhinol Laryngol 1997;106:109-116.

7. Brandt MG, Wright ED. The silent sinus syndrome is a form of chronic maxillary atelectasis: a systematic review of all reported cases. Am J Rhinol 2008 Jan-Feb;22(1):68-73.

8. Hourany R, Aygun N, Charley C. Santina D, Zinreich SJ. Silent sinus syndrome: an acquired condition. Am J Neuroradiol 2005 Oct;26:2390-2392.

9. Kruger TL, Myint SA. Silent sinus syndrome and scuba diving: case report and review and literature review. Clinical and Surgical Ophthalmology 30(1);18-22.

10. Davidson JK, Soparkar CNS, Williams JB, Patrinely JR. Negative sinus pressure and normal predisease imaging in the silent sinus syndrome. Arch Ophthalmol 1999;117:1653-1654.

11. Levine SB, Mitra S. Maxillary sinus involution after endoscopic sinus surgery in a child: a case report. Am J Rhinol 2000;14: 7-11.

12. Naik RN, Khemani S, Saleh HA. Frontal silent sinus syndrome Otolaryngology, Head and Neck Surgery 148(2);354-355.

\section{ABOUT THE AUTHORS}

\section{Marina Saldanha}

Assistant Professor, Department of Otorhinolaryngology, KS Hegde Medical Academy, Mangalore, Karnataka, India

\section{Vadisha Bhat (Corresponding Author)}

Associate Professor, Department of Otorhinolaryngology, KS Hegde Medical Academy, Mangalore, Karnataka, India, Phone: +91-9480174828, e-mail: bvadish@yahoo.co.in

\section{B Satheesh Kumar Bhandary}

Professor, Department of Otorhinolaryngology, KS Hegde Medical Academy, Mangalore, Karnataka, India

\section{Sebin Tom Scaria}

Resident, Department of Otorhinolaryngology, KS Hegde Medical Academy, Mangalore, Karnataka, India 